\title{
SBH10: A Benchmark Database of Barrier Heights on Transition Metal Surfaces
}

Shaama Mallikarjun Sharada, ${ }^{\dagger}$ Thomas Bligaard, ${ }^{\dagger}$ Alan C. Luntz, ${ }^{\dagger}$ Geert-Jan Kroes, ${ }^{\ddagger}$ and Jens K. Nørskov*, ${ }^{\dagger}$

${ }^{\dagger}$ Department of Chemical Engineering, Stanford University, 443 Via Ortega, Stanford California 94305, United States

${ }^{\ddagger}$ Leiden Institute of Chemistry, Gorlaeus Laboratories, Leiden University, P.O. Box 9502, 2300 RA Leiden, The Netherlands

Supporting Information

ABSTRACT: While the performance of density functional approximations (DFAs) for gas phase reaction energetics has been extensively benchmarked, their reliability for activation barriers on surfaces is not fully understood. The primary reason for this is the absence of well-defined, chemically accurate benchmark databases for chemistry on surfaces. We present a database of 10 surface barrier heights for dissociation of small molecules, SBH10, based on carefully chosen references from molecular beam scattering, laser assisted associative desorption, and thermal experiments. Our benchmarking study compares the performance of a dispersion-corrected generalized gradient approximation (GGA-vdW), BEEF-vdW, a meta-GGA, MS2, and a screened hybrid functional, HSE06. In stark contrast to gas phase reactions for which GGAs systematically underestimate barrier heights and hybrids tend to be most accurate, the BEEF-vdW functional determines barriers accurately to within $0.14 \mathrm{eV}$ of experiments, while MS2 and HSE06 underestimate barrier heights on surfaces. Higher accuracy of BEEF-vdW stems from the fact that the functional is trained on chemisorption systems, and transition states for dissociation on surfaces closely resemble the final, chemisorbed states. Therefore, a functional that can describe chemisorption accurately can also reliably predict barrier heights on surfaces.

\section{INTRODUCTION}

Kohn-Sham density functional theory (KS-DFT) ${ }^{1,2}$ is an invaluable tool for enhancing our understanding of moleculesurface interactions. DFT simulations have found widespread applications in the fields of heterogeneous catalysis, ${ }^{3}$ electrochemistry, ${ }^{4}$ spectroscopy, ${ }^{5}$ and energy storage, ${ }^{6}$ to name a few. Generalized gradient approximations (GGAs) have shown some success in the description of adsorption on transition metal surfaces. ${ }^{7-10}$ However, kinetics studies also demand accurate barrier heights, for which little is known about the appropriate choice of functional or functional class (GGA, meta-GGA, hybrid functionals). ${ }^{11}$ For reactions occurring in the gas phase, it is well-known that GGAs underestimate barrier heights as a consequence of self-interaction errors. ${ }^{12}$ These errors arise from incomplete cancellation of Coulomb and exchange interactions of an electron with itself. ${ }^{13}$ Hybrid functionals, which include a fraction of exact (Hartree-Fock) exchange, can partially correct for self-interaction errors and improve activation barrier estimates. ${ }^{14,15}$ Extensive benchmarking studies of functional accuracies for reactions in the gas phase have been made possible by the availability of several high accuracy barrier heights databases ${ }^{16,17}$ calculated using the $\operatorname{CCSD}(\mathrm{T})^{18}$ "gold standard". Such benchmarks are not available for reactions on surfaces owing to the prohibitive cost of $\operatorname{CCSD}(\mathrm{T})$ for periodic systems. Therefore, we have to turn to single-crystal experiments that examine the dissociation of small molecules on well-defined surfaces in order to collate a database of benchmark barrier heights.

We propose the construction of such a database, consisting of accurate barriers for dissociation reactions of molecules on transition metal surfaces. The references are chosen from three types of experiments-molecular beam experiments combined with quantum dynamics studies, laser assisted associative desorption, and thermal rate measurements. The use of combined molecular beam experiments and $a b$ initio dynamics to obtain benchmark barriers on surfaces was proposed recently. ${ }^{19}$ In molecular beam experiments, gas molecules of a particular rotational and vibrational configuration collide with the surface of a metal, and are either scattered or dissociated as a result. The dynamics of dissociation are then examined by mapping the reaction probability as a function of collision energy. ${ }^{20}$ Like quasi-classical trajectory studies, quantum dynamics techniques, which can be used to study $\mathrm{H}_{2}$ dissociation on metals while taking into account motion in all molecular degrees of freedom, aim to reproduce experimental probability curves by simulating the dissociation phenomena from static DFT-based potential energy surfaces. ${ }^{21}$ The barriers determined in these dynamics studies, which accurately

Received: June 9, 2017

Revised: July 30, 2017

Published: August 3, 2017 
reproduce experimental reactive probabilities, can serve as accurate benchmarks for DFT performance.

The second type of study, laser assisted associative desorption (LAAD), experimentally probes the dynamics of the reverse of dissociative adsorption on transition metal surfaces. $^{22}$ A laser beam is employed to induce associative desorption of atoms adsorbed on a surface. The highest kinetic energy of the desorbing molecules, when extrapolated to zero surface temperature, corresponds to the lowest adiabatic barrier.

The third technique involves thermal rate measurements of dissociative adsorption on single crystal transition metal surfaces, which determine activation barriers from Arrhenius curves plotted over the experimental temperature range. While thermal experiments offer the most direct means of determining adiabatic barriers, they are highly sensitive to the presence of impurities and defects on the transition metal surface since the kinetics are often several orders of magnitude faster at these sites. ${ }^{23}$ Therefore, thermal experiments provide accurate references for reactions occurring at defects, while molecular beam and LAAD experiments are more suited to reactions occurring on terrace sites of transition metal surfaces.

Our objective is to construct an accurate database of barrier heights on transition metal surfaces for the purposes of developing and benchmarking density functionals. The reference barriers are carefully chosen from molecular beam, $\mathrm{LAAD}$, and thermal experiments. As a first step toward understanding accuracies and systematic trends in barrier height prediction, we calculate barriers for representative GGA$\mathrm{vdW}$, meta-GGA, and screened hybrid functionals.

\section{PROCEDURE}

2.1. Density Functional Approximations. The Vasp 5.3.5 simulation package is employed for all calculations. ${ }^{24-27}$ The details of model setups and barrier height calculations are presented in the Supporting Information. Three functionals are chosen for benchmarking DFT accuracies for the prediction of barrier heights. Benchmarking studies on commonly used GGAs and dispersion-corrected GGAs in surface chemistry have demonstrated that the BEEF-vdW ${ }^{8}$ functional yields the lowest errors for chemisorption energies on transition metal surfaces. 9,28 This is because the exchange correlation parameters in BEEF-vdW have been trained on several properties relevant to surface chemistry. Therefore, BEEF$\mathrm{vdW}$ is chosen to represent the second rung (GGA) of Jacob's ladder. ${ }^{11}$ Although the functional consists of a nonlocal method for calculating dispersion contributions, vdW-DF2, ${ }^{29}$ this term can be evaluated efficiently. ${ }^{30}$ The MS2 functional ${ }^{31}$ is chosen as the representative meta-GGA (third rung) functional, since it improves description of bulk properties relative to GGAs. The application of hybrid functionals to surface chemistry has been limited due to both high computational costs as well as the poor description of metallic systems with Hartree-Fock exchange. $^{32}$ Nevertheless, short-range screened hybrid functionals, like the HSE family, have been successfully employed in studying adsorption on transition metal surfaces. ${ }^{33,34}$ Therefore, in order to examine the accuracy of the fourth rung of Jacob's ladder, ${ }^{11}$ we choose the short-range screened hybrid HSE06 functional. ${ }^{35}$

Transition state energies $\left(E_{T S}\right)$, or barriers, are determined as the zero-point corrected energy difference between the transition state and the isolated gas phase molecule, as shown in Scheme 1. Barriers calculated with BEEF-vdW, MS2, and
Scheme 1. Reaction Path Schematic for Dissociative Chemisorption on Transition Metal Surfaces ${ }^{a}$

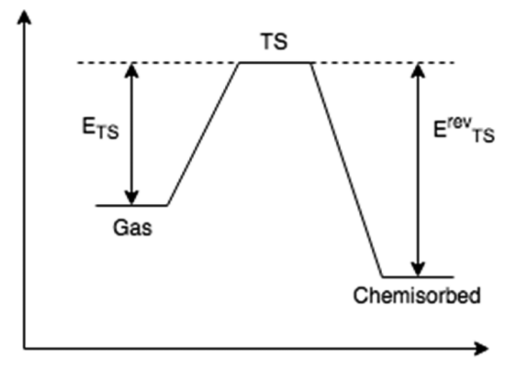

${ }^{a}$ Barriers $\left(E_{T S}\right)$ are calculated as the difference between the transition state energy on the surface and the corresponding gas phase reference. We also compare BEEF-vdW and MS2 barriers for the reverse reaction $\left(E_{T S}^{r e v}\right)$, associative desorption.

HSE06 functionals are benchmarked against experimental references for dissociation reactions in the barrier heights database described below. Owing to the prohibitive cost associated with transition state search and optimization with hybrid functionals, we determine HSE06 barriers only for the four reactions of the smallest dissociating molecule, $\mathrm{H}_{2}$. Since HSE06 errors for $\mathrm{H}_{2}$ dissociation barriers are very similar to MS2 errors, we believe that the analysis of MS2 performance can be extended to HSE06 as well.

2.2. Benchmark Database of 10 Surface Barrier Heights: SBH10. The reactions constituting the SBH10 benchmark database of barrier heights on surfaces are listed in Table 1, along with the chosen reference barriers and the

Table 1. SBH10 Database of Experimentally Observed Reference Barrier Heights (eV) for Dissociation Reactions on Transition Metal Surfaces

\begin{tabular}{|c|c|c|c|}
\hline & dissociation reaction, site type & experiment & barrier $(\mathrm{eV})$ \\
\hline 1 & $\mathrm{H}_{2} / \mathrm{Cu}(111)$, terrace & molecular beam ${ }^{65,66,19}$ & 0.63 \\
\hline 2 & $\mathrm{H}_{2} / \mathrm{Cu}(100)$, terrace & molecular beam ${ }^{67,19}$ & 0.74 \\
\hline 3 & $\mathrm{H}_{2} / \mathrm{Pt}(111)$, terrace & molecular beam ${ }^{68,45,46}$ & 0.00 \\
\hline 4 & $\mathrm{H}_{2} / \mathrm{Ru}(0001)$, terrace & molecular beam ${ }^{69,43}$ & 0.00 \\
\hline 5 & $\mathrm{~N}_{2} / \mathrm{Ru}(0001)$, terrace & molecular beam ${ }^{70,51}$ & 1.84 \\
\hline 6 & $\mathrm{~N}_{2} / \mathrm{Ru}(0001)$, step & thermal $^{23}$ & 0.40 \\
\hline 7 & $\mathrm{CH}_{4} / \mathrm{Ru}(0001)$, terrace & $\mathrm{LAAD}^{60}$ & 0.80 \\
\hline 8 & $\mathrm{CH}_{4} / \mathrm{Ni}(100)$, terrace & molecular beam ${ }^{58}$ & 0.76 \\
\hline 9 & $\mathrm{CH}_{4} / \mathrm{Ni}(111)$, terrace & molecular beam $^{55}$ & 1.01 \\
\hline 10 & $\mathrm{CH}_{4} / \mathrm{Ni}(111)$, step & thermal $^{56}$ & 0.80 \\
\hline
\end{tabular}

final adsorption sites of the dissociated species. We describe the selection procedure for experimental benchmarks for dissociation reactions of $\mathrm{H}_{2}, \mathrm{~N}_{2}$, and $\mathrm{CH}_{4}$. We also justify the choice of experimental reference in situations where multiple, and sometimes conflicting barrier heights are reported. Although a single reference value is chosen for each reaction, we account for the range in reported barriers by estimating experimental uncertainties (error bars) in our analysis.

2.2.1. $\mathrm{H}_{2}$ Dissociation on $\mathrm{Cu}(111), \mathrm{Cu}(100), \mathrm{Pt}(111)$, and $\mathrm{Ru}(0001)$ Terraces. The dissociation of $\mathrm{H}_{2}$ on $\mathrm{Cu}$ is one of the most widely studied systems in the context of activated dissociative adsorption for applications in catalysis and corrosion. $^{36,37}$ These reactions are ideal benchmarks for electronic structure methods since an adiabatic potential energy surface approximation, in the absence of electron-hole pair 
Table 2. Comparison between Experimental Reference and Calculated (Zero-Point Corrected) $E_{\mathrm{TS}}$ Using BEEF-vdW, MS2, and HSE06 Functionals ${ }^{a}$

\begin{tabular}{|c|c|c|c|c|c|c|}
\hline & \multirow[b]{2}{*}{ dissociation reaction, site type } & \multirow[b]{2}{*}{ barrier, reference $(\mathrm{eV})$} & \multirow[b]{2}{*}{ zero-point correction $(\mathrm{eV})$} & \multicolumn{3}{|c|}{$E_{\mathrm{TS}}, \mathrm{DFT}(\mathrm{eV})$} \\
\hline & & & & BEEF-vdW & MS2 & HSE06 \\
\hline 1 & $\mathrm{H}_{2} / \mathrm{Cu}(111)$, terrace & 0.63 & -0.06 & 0.71 & 0.29 & 0.20 \\
\hline 2 & $\mathrm{H}_{2} / \mathrm{Cu}(100)$, terrace & 0.74 & -0.08 & 0.72 & 0.34 & 0.58 \\
\hline 3 & $\mathrm{H}_{2} / \mathrm{Pt}(111)$, terrace & 0 & 0.00 & 0.12 & -0.32 & -0.44 \\
\hline 4 & $\mathrm{H}_{2} / \mathrm{Ru}(0001)$, terrace & 0 & -0.03 & -0.16 & -0.54 & -0.69 \\
\hline 5 & $\mathrm{~N}_{2} / \mathrm{Ru}(0001)$, terrace & 1.84 & -0.04 & 1.61 & 1.02 & - \\
\hline 6 & $\mathrm{~N}_{2} / \mathrm{Ru}(0001)$, step & 0.40 & -0.05 & 0.60 & 0.18 & - \\
\hline 7 & $\mathrm{CH}_{4} / \mathrm{Ru}(0001)$, terrace & 0.80 & -0.11 & 0.82 & 0.71 & - \\
\hline 8 & $\mathrm{CH}_{4} / \mathrm{Ni}(100)$, terrace & 0.76 & -0.12 & 0.90 & - & - \\
\hline 9 & $\mathrm{CH}_{4} / \mathrm{Ni}(111)$, terrace & 1.01 & -0.12 & 1.14 & 1.06 & - \\
\hline 10 & $\mathrm{CH}_{4} / \mathrm{Ni}(111)$, step & 0.80 & -0.14 & 0.84 & 0.38 & - \\
\hline
\end{tabular}

${ }^{a}$ BEEF-vdW zero-point energy corrections are reported. The transition state geometry for $\mathrm{CH}_{4}$ dissociation on $\mathrm{Ni}(100)$ could not be calculated with the MS2 functional using either fixed bond length or dimer methods, possibly due to the shallow nature of the potential energy surface close to the transition state.

excitations and phonon interactions, provides an accurate description of the dissociating system. ${ }^{38,39,37}$

Experimental reactive scattering probabilities of $\mathrm{H}_{2}$ dissociation on $\mathrm{Cu}(111)$ and $\mathrm{Cu}(100)$ have been successfully reproduced using full-dimensional quantum dynamics methods, based on potential energy surfaces calculated using the specific reaction parameter approach to density functional theory (SRPDFT). ${ }^{21,36,40}$ Therefore, we employ these barriers, 0.63 and $0.74 \mathrm{eV}$ for $\mathrm{Cu}(111)$ and $\mathrm{Cu}(100)$, respectively, as references for $\mathrm{H}_{2}$ dissociation on $\mathrm{Cu}^{19}$ Rasmussen et al. carried out thermal rate measurements for $\mathrm{H}_{2}$ dissociation on $\mathrm{Cu}(100)$, and reported a lower barrier, $0.5 \mathrm{eV} .^{41}$ There is a noticeable curvature at high temperatures in the Arrhenius relationship described in Figure 2 of their work. ${ }^{41}$ Using the low temperature results in this data, we recalculate the barrier to compare more directly with our adiabatic electronic structure calculations. The barrier at the low temperature limit is $0.6 \mathrm{eV}$. This result is also in agreement with thermal measurements of $\mathrm{H}_{2}$ dissociation on $\mathrm{Cu}(100)$, which determine a barrier of 0.62 $\mathrm{eV}^{42}$ While the reactive scattering barrier is employed as reference for $\mathrm{H}_{2} / \mathrm{Cu}(100)$, both scattering and thermal barriers are used to estimate the uncertainty in experiments.

In computational scattering studies of $\mathrm{H}_{2}$ dissociation on $\mathrm{Ru}(0001)$, reaction probabilities from DFT potential energy surfaces obtained with DFAs of the GGA-vdW type yield good agreement with experiments. ${ }^{43}$ This is a weakly activated process, and barriers determined using both dynamics $(\approx 4$ $\mathrm{meV})^{43}$ and $\mathrm{LAAD}^{44}$ are close to zero. $\mathrm{H}_{2}$ is also weakly activated on $\mathrm{Pt}(111)$ with minimum barrier heights of 0.06 $\mathrm{eV}^{45}$ and $-8 \mathrm{meV}^{46}$ determined from quantum dynamics comparison to beam experiments. Therefore, the reference barriers for the dissociation of $\mathrm{H}_{2}$ on $\mathrm{Ru}(0001)$ and $\mathrm{Pt}(111)$ are both set to $0 \mathrm{eV}$.

2.2.2. $\mathrm{N}_{2}$ Dissociation on $\mathrm{Ru}(0001)$ Terrace and Step. The best catalysts for the Haber-Bosch process for the synthesis of ammonia are ruthenium-based. ${ }^{47}$ Since the dissociation of $\mathrm{N}_{2}$ is the rate-limiting step on $\mathrm{Ru}$ catalysts, it has been the subject of several single crystal experiments. Thermal experiments by Dahl et al. ${ }^{23,48}$ have demonstrated that the dissociative chemisorption of $\mathrm{N}_{2}$ is completely dominated by step sites, over which the barrier is $0.4 \mathrm{eV}$.

The barriers reported for $\mathrm{N}_{2}$ dissociation on $\mathrm{Ru}(0001)$ terrace sites vary widely depending on the underlying experimental technique. While thermal experiments ${ }^{4,49}$ report a barrier of $1.3 \mathrm{eV}$, it is difficult to deduce whether this is a consequence of $\mathrm{N}_{2}$ dissociation on terraces or displacement of $\mathrm{Au}$ atoms that were used to block the step sites. LAAD experiments, carried out using a $\mathrm{Ru}(0001)$ surface that is already covered with chemisorbed $\mathrm{N}$, determine the barrier at the lowest $\mathrm{N}$ precoverage $(0.23)$ to be $1.8 \mathrm{eV} .^{22}$ Dissociation curves determined using quasi-classical dynamics using a barrier of $2.27 \mathrm{eV}$ are in qualitative agreement with molecular beam experiments. ${ }^{50}$ Quantitative agreement between scattering experiments and theory is achieved with a barrier of $1.84 \mathrm{eV}$ in a more recent dynamics study that uses a neural network potential model of the potential energy surface. ${ }^{51} \mathrm{We}$ employ this barrier as the reference for $\mathrm{N}_{2}$ dissociation on $\mathrm{Ru}(0001)$ terrace sites.

2.2.3. $\mathrm{CH}_{4}$ Dissociation on Ni(111) Terrace and Step, $\mathrm{Ni}(100)$ and $\mathrm{Ru}(0001)$ Terraces. The dissociation dynamics of $\mathrm{CH}_{4}$ on $\mathrm{Ni}$ and $\mathrm{Ru}$ catalysts are of significant interest to industry since dissociation constitutes the first step in the steam reforming of natural gas to produce syngas for alcohol synthesis and the Fischer-Tropsch process. Activation energies for $\mathrm{CH}_{4}$ dissociation are highly sensitive to experimental conditions, including gas and surface temperatures and pressures. ${ }^{38,52,57}$ Since the barrier heights are also strongly coupled to the motion of the lattice, dynamics studies that account for lattice coupling in their models are desirable as references. ${ }^{53}$

Thermal experiments report a barrier of $0.77 \mathrm{eV}$ on $\mathrm{Ni}(111){ }^{54}$ while molecular beam studies combined with SRP-DFT and lattice-coupled dynamics report $1.01 \mathrm{eV}^{55}$ Interestingly, the difference between molecular beam and thermal barriers corresponds almost exactly to the difference between terrace and step site barriers, $0.21 \mathrm{eV}$, determined by a combined experimental and theoretical study. ${ }^{56}$ Therefore, we use $1.01 \mathrm{eV}$ as the reference for $\mathrm{Ni}(111)$ terrace sites and 0.8 $\mathrm{eV}(=1.01-0.21 \mathrm{eV})$ as the reference for $\mathrm{Ni}(111)$ step sites. Similar differences are observed for $\mathrm{Ni}(100)$ where thermal experiments $^{57}$ and molecular beam dynamics ${ }^{58}$ report 0.61 and $0.76 \mathrm{eV}$, respectively. We employ the latter as reference for $\mathrm{CH}_{4}$ dissociation on $\mathrm{Ni}(100)$ terrace sites.

For dissociation of $\mathrm{CH}_{4}$ on $\mathrm{Ru}(0001)$, thermal, molecular beam, and LAAD studies report barrier heights of $0.53,{ }^{54}$ $0.38,{ }^{59}$ and $0.8 \mathrm{eV}{ }^{60}$ respectively. The difference between $\mathrm{LAAD}$ and thermal barriers are similar to the difference observed between terrace and step sites on $\mathrm{Ni}(111)$. The barrier determined for the dynamics model with molecular 
beam experiments is not entirely reliable since it assumes a simplified quasi-diatomic $2 \mathrm{D}$ system and neglects lattice motion. Mortensen et al. ${ }^{60}$ report the results of both molecular beam dissociation as well as LAAD experiments in order to examine dissociation from both sides of the reaction barrier. The molecular beam barrier for dissociative adsorption obtained indirectly from calculation of the sticking threshold, $0.85 \mathrm{eV}$, is in good agreement with the LAAD adiabatic barrier, $0.8 \mathrm{eV}$. Therefore, we employ the LAAD barrier as reference for methane dissociation on $\mathrm{Ru}(0001)$ terrace sites.

We acknowledge that this database is not comprehensive. There are systems which are excluded owing to difficulties in either interpreting experiments or determining the model surface for dissociation. For instance, dissociation of $\mathrm{CO}$ on step sites of $\mathrm{Ni}$ is not included since it has been shown that barriers are highly sensitive to both the choice of adsorption site as well as coverage. ${ }^{61}$ However, there is ample scope for expansion of the database of barrier heights, to include dissociation of $\mathrm{CH}_{4}$ on $\mathrm{Pt}(111)$ and $\mathrm{Pt}(211),{ }^{62} \mathrm{NH}_{3}$ on $\mathrm{Ru}(0001),{ }^{63}$ and $\mathrm{H}_{2} \mathrm{O}$ on $\mathrm{Ni}(111){ }^{64}$

\section{RESULTS AND DISCUSSION}

3.1. SBH10 - Benchmarking Functionals. Table 2 summarizes the results of functional benchmarking for the SBH10 database, and Table 3 reports errors and error statistics.

Table 3. SBH10 errors for BEEF-vdW, MS2 and HSE06 functionals. $\sigma_{\mathrm{BEE}}$ corresponds to the scaled Bayesian error estimate for the BEEF-vdW functional. The average error statistics are also reported

\begin{tabular}{|c|c|c|c|c|c|}
\hline & \multirow[b]{2}{*}{$\begin{array}{l}\text { dissociation reaction, site } \\
\text { type }\end{array}$} & \multicolumn{4}{|c|}{ errors, DFT - experiment (eV) } \\
\hline & & $\begin{array}{l}\text { BEEF- } \\
\text { vdW }\end{array}$ & $\begin{array}{l}\sigma_{\mathrm{BEE}} \\
\text { scaled }\end{array}$ & MS2 & HSE06 \\
\hline 1 & $\mathrm{H}_{2} / \mathrm{Cu}(111)$, terrace & 0.08 & 0.15 & -0.34 & -0.43 \\
\hline 2 & $\mathrm{H}_{2} / \mathrm{Cu}(100)$, terrace & -0.02 & 0.16 & -0.40 & -0.16 \\
\hline 3 & $\mathrm{H}_{2} / \mathrm{Pt}(111)$, terrace & 0.12 & 0.13 & -0.32 & -0.44 \\
\hline 4 & $\mathrm{H}_{2} / \mathrm{Ru}(0001)$, terrace & -0.16 & 0.12 & -0.54 & -0.69 \\
\hline 5 & $\mathrm{~N}_{2} / \mathrm{Ru}(0001)$, terrace & -0.23 & 0.26 & -0.82 & - \\
\hline 6 & $\mathrm{~N}_{2} / \mathrm{Ru}(0001)$, step & 0.20 & 0.32 & -0.22 & - \\
\hline 7 & $\mathrm{CH}_{4} / \mathrm{Ru}(0001)$, terrace & 0.02 & 0.13 & -0.09 & - \\
\hline 8 & $\mathrm{CH}_{4} / \mathrm{Ni}(100)$, terrace & 0.14 & 0.16 & - & - \\
\hline 9 & $\mathrm{CH}_{4} / \mathrm{Ni}(111)$, terrace & 0.13 & 0.14 & 0.05 & - \\
\hline \multirow[t]{4}{*}{10} & $\mathrm{CH}_{4} / \mathrm{Ni}(111)$, step & 0.04 & 0.18 & -0.42 & - \\
\hline & mean signed error, MSE, eV & 0.03 & 0.17 & -0.34 & -0.43 \\
\hline & $\begin{array}{l}\text { mean absolute error, } \\
\text { MAE, eV }\end{array}$ & 0.12 & & 0.36 & 0.43 \\
\hline & $\begin{array}{l}\text { root mean square } \\
\text { error, RMSE, eV }\end{array}$ & 0.14 & & 0.42 & 0.47 \\
\hline
\end{tabular}

The parity plots between experiment and theory for BEEFvdW, MS2, and HSE06 functionals, are depicted in Figure 1. In cases where several experimental barriers are reported, the variances are depicted as experimental (horizontal) error bars.

Scattering experiments coupled with quantum dynamics studies predict essentially barrierless dissociation for $\mathrm{H}_{2}$ on $\mathrm{Ru}(0001)$ and $\mathrm{Pt}(111)$ surfaces. However, DFT calculations sometimes predict negative $E_{T S}$ for these reactions. Since this is in direct contradiction with the measurement of sizable diffraction probabilities for both systems, ${ }^{43,71}$ the large negative barriers resulting mostly from MS2 and HSE06 calculations are erroneous. The gray arrows in Figure 1 correspond to these results and indicate that the functional estimates an unphysical
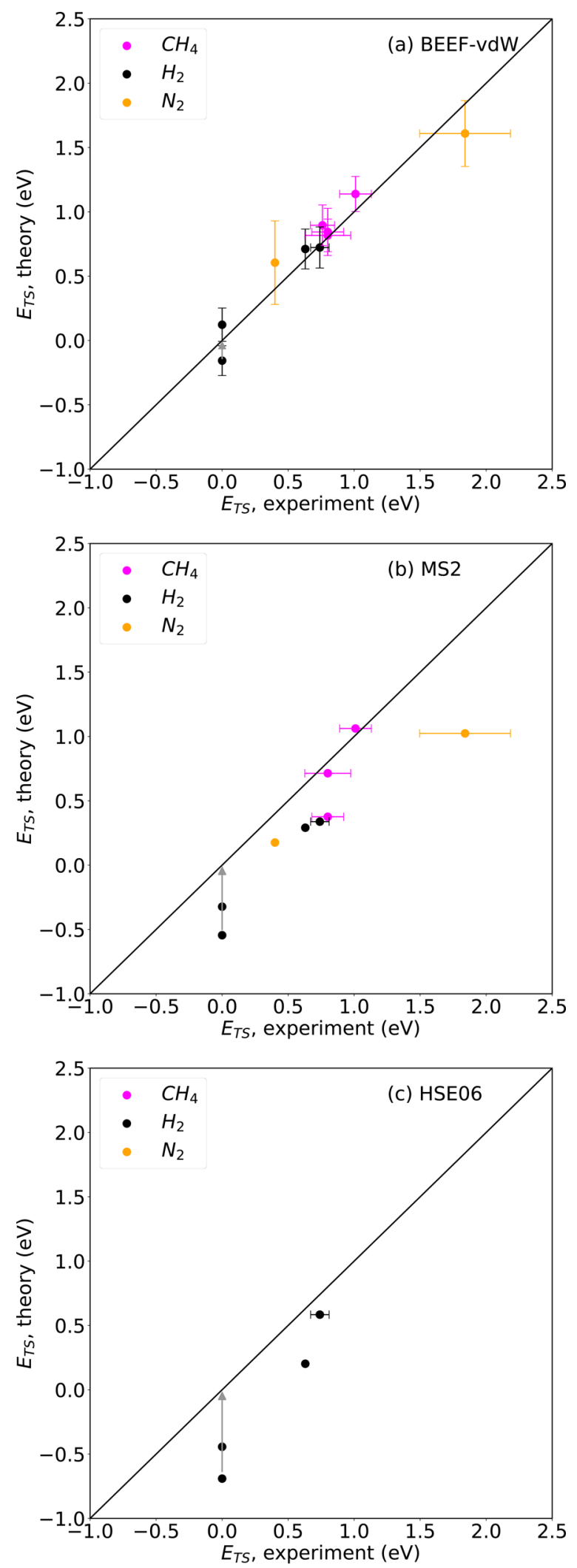

Figure 1. Parity plots benchmarking the accuracies of DFA's for transition state energies $\left(E_{T S}\right)$ constituting the SBH10 database. (a) BEEF-vdW, (b) MS2, and (c) HSE06 functionals. The gray arrows, when present, indicate that the functional estimates an unphysical negative barrier, which should, in principle, lie on the parity line. 
negative barrier, which should, in principle, lie on the parity line.

3.1.1. BEEF-vdW. GGAs and meta-GGAs severely underestimate barrier heights in gas phase reactions owing to selfinteraction errors that lead to incorrect charge separation at the transition state. ${ }^{31}$ Hybrid functionals, by virtue of including a fraction of exact exchange, alleviate the problem of selfinteraction and improve the description of the transition state. $^{72}$

The trends are reversed for reactions occurring on transition metal surfaces. BEEF-vdW energies are in excellent agreement with experiments, exhibiting the lowest errors among the three functionals. The root-mean-square error (RMSE) for BEEF$\mathrm{vdW}$ is $0.14 \mathrm{eV}$, demonstrating that this is an excellent functional for estimating barriers. In contrast, gas phase barriers with BEEF-vdW are systematically underestimated, with an RMSE of $0.33 \mathrm{eV}^{8}$ The Bayesian error estimates (BEE) determined with the BEEF-vdW functional are denoted by $\sigma_{\mathrm{BEE}}$ in Table 3. The error estimates represent the uncertainty in calculated barriers arising from the choice of BEEF-vdW exchange correlation model parameters, scaled by a factor of 2 / 3 to reflect uncertainties in the prediction of chemisorption on transition metal surfaces. ${ }^{73}$ It must be noted that these uncertainties do not take into account errors arising from incompleteness of the exchange correlation model. $\sigma_{\mathrm{BEE}}$ values are also plotted as y error bars in Figure 1a. In general, the error estimates are of the right order of magnitude, and in line with those determined in a benchmarking study of chemisorption energies on transition metal surfaces. ' The BEEF-vdW transition state geometries are presented as Vasp POSCAR files in the Supporting Information.

3.1.2. MS2 and HSEO6. As seen in Tables 2 and 3 and Figure 1, parts b and c, MS2 and HSE06 functionals typically underestimate transition state energies, with negative mean signed errors (MSE) of -0.34 and $-0.43 \mathrm{eV}$, respectively. On average, $E_{\mathrm{TS}}$ errors for $\mathrm{H}_{2}$ dissociation with HSE06 are similar for MS2 $(-0.4 \mathrm{eV})$. Therefore, we believe that MS2 errors and trends for the remaining systems in SBH10 are transferable to HSE06 as well. MS2 accuracies also appear to be systemdependent, since the functional exhibits lower errors for some $\mathrm{CH}_{4}$ dissociation reactions relative to $\mathrm{H}_{2}$ and $\mathrm{N}_{2}$. In the following sections, we examine the reasons for the superior performance of the GGA-vdW functional relative to metaGGA and screened hybrids.

All three functionals in this study predict negative $E_{T S}$ for $\mathrm{H}_{2}$ dissociation on $\mathrm{Ru}(0001)$. The calculated transition state geometry for this reaction corresponds to the later of the two possible transition states determined at the top site by Wijzenbroek and Kroes in Figure 2 of their paper. ${ }^{43}$ They have demonstrated that, depending on the functional, the two transition states can differ in energies by up to $0.64 \mathrm{eV}$. This uncertainty in transition state geometry, therefore, may also be contributing to $E_{T S}$ errors for $\mathrm{H}_{2}$ dissociating on $\mathrm{Ru}(0001)$.

3.2. Analysis of Barrier Prediction Accuracies. 3.2.1. Associative Desorption. In addition to dissociative adsorption, we compare barriers to the reverse process, associative desorption. The BEEF-vdW and MS2 barriers, given by the difference between electronic energies of the transition state and dissociated final state, are shown in Figure 2. In general, the difference between MS2 and BEEF-vdW barriers to associative desorption are much smaller than dissociative adsorption. Similar intrinsic barriers for associative desorption across functionals indicates that the differences

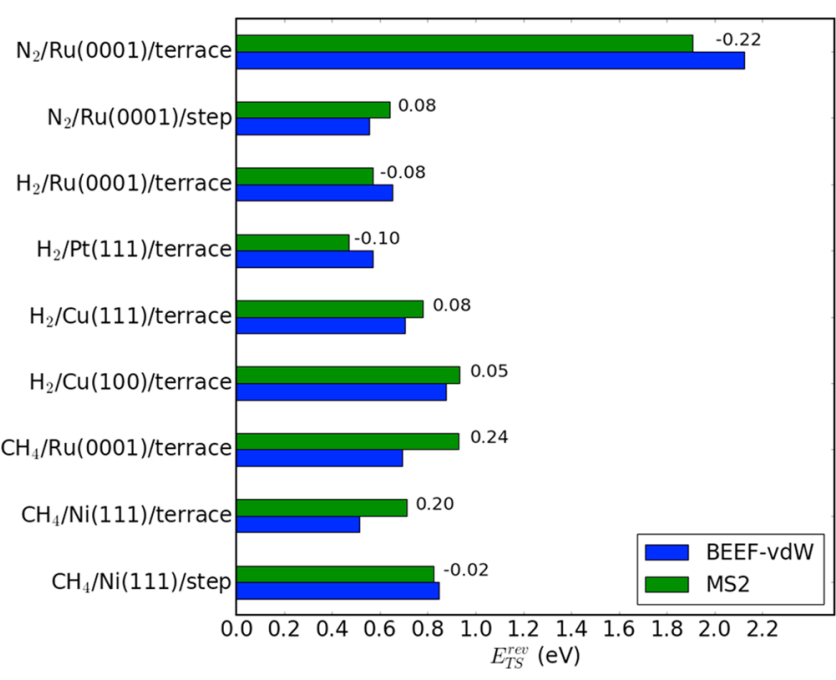

Figure 2. BEEF-vdW and MS2 barrier heights for the reverse reaction, associative desorption $\left(E_{\mathrm{TS}}^{\mathrm{rev}}\right)(\mathrm{eV})$. The difference between MS2 and BEEF-vdW barriers are also reported for each reaction.

arising from varying description of the bulk and surface properties of the transition metal (such as lattice constants or density of states) neatly cancel out. It also suggests that the theoretical description of the transition state closely follows that of the final state. In other words, the performance of a functional for barrier heights prediction is reflected in its accuracy in predicting chemisorption energies.

3.2.2. Chemisorption Energies. Since dissociation reactions typically occur via transition states that are more final state-like, errors in $\mathrm{E}_{\mathrm{TS}}$ must be very similar to errors in chemisorption energies. This hypothesis is difficult to test since, with the exception of $\mathrm{H}_{2}$ dissociation on $\mathrm{Pt}(111)$ terraces, there is no overlap between the systems constituting $\mathrm{SBH} 10$ and the systems for which high accuracy single-crystal experimental chemisorption energies are available (CE39/ADS41). ${ }^{9,74}$ Nevertheless, we compare both the average errors between SBH10 and 26 chemisorption systems (CE26) in the adsorption database, ADS41, as well as systems specific to $\mathrm{H}_{2}$ dissociation.

The comparison between error statistics for the two databases is presented in Table 4. Since the number of chemisorbed species produced by the different surface reactions in the CE26 database can vary between 1 and 3, CE26 errors are reported on a per adsorbate basis. It is evident that BEEF$\mathrm{vdW}$ is the best-performing functional, as it predicts both barrier heights and chemisorption energies to within $0.2 \mathrm{eV}$. On the other hand, MS2 and HSE06 both overbind chemisorbed atoms and molecules, and as a result, underestimate barrier heights on transition metal surfaces.

Figure 3 is an illustrative example of these trends for dissociative chemisorption of $\mathrm{H}_{2}$. The $\mathrm{H}_{2}$ dissociation reactions in the $\mathrm{SBH} 10$ database are compared with the $\mathrm{H}$ chemisorption systems in the ADS41 database. BEEF-vdW underbinds $\mathrm{H}$ on transition metal surfaces, which possibly compensates for selfinteraction errors that tend to lower transition state energies, thereby leading to accurate barriers. In the case of $\mathrm{H}_{2} / \mathrm{Pt}(111)$, for which both barriers and chemisorption references are available, this underbinding leads to overestimation of both the chemisorption energy and barrier by 0.28 and $0.12 \mathrm{eV}$, respectively. On the other hand, MS2 and HSE06 both 
Table 4. Comparison ${ }^{a}$ between Error Statistics (eV) across the Three Functionals (BEEF-vdW, MS2, HSE06) for Barrier Heights in SBH10 and 26 Chemisorption Systems (CE26) in the ADS41 Database ${ }^{74}$

\begin{tabular}{|c|c|c|c|c|c|c|}
\hline \multirow[b]{2}{*}{ DFA } & \multicolumn{3}{|c|}{ SBH10 } & \multicolumn{3}{|c|}{ CE26 } \\
\hline & MSE & MAE & RMSE & MSE & MAE & RMSE \\
\hline BEEF-vdW & $0.03(0.02)$ & $0.12(0.06)$ & $0.14(0.07)$ & -0.03 & 0.17 & 0.20 \\
\hline MS2 & $-0.34(-0.17)$ & $0.36(0.18)$ & $0.42(0.21)$ & -0.15 & 0.23 & 0.27 \\
\hline HSE06 & $-0.43(-0.21)$ & $0.43(0.21)$ & $0.47(0.23)$ & -0.23 & 0.34 & 0.41 \\
\hline
\end{tabular}

${ }^{a}$ Values in parentheses represent errors scaled to a per fragment basis for the SBH10 database.
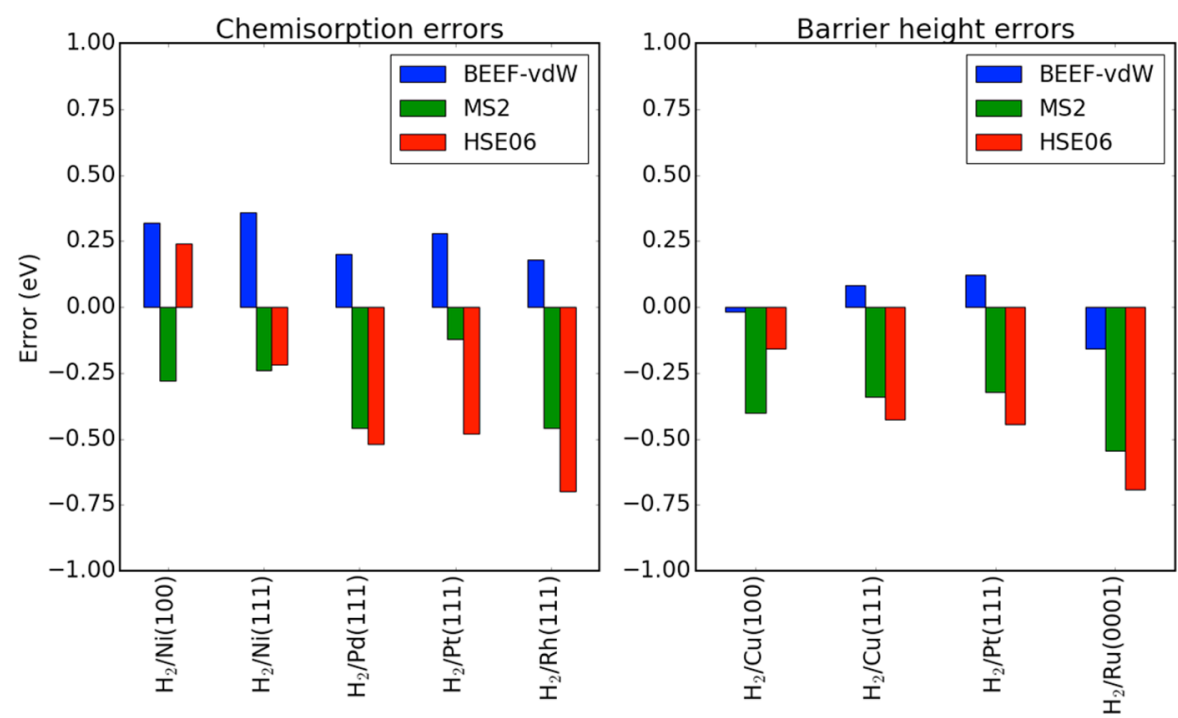

Figure 3. Comparison between functional errors $(\mathrm{eV})$ in the prediction of $\mathrm{H}$ chemisorption energies in the ADS41 database and barrier heights for $\mathrm{H}_{2}$ dissociation reactions in the SBH10 database. Chemisorption energy errors (per adsorbate) are scaled by a factor of 2 for appropriate comparison with barrier heights.

overbind $\mathrm{H}$, and they predict more negative chemisorption energies and hence smaller barrier heights.

Therefore, barrier height accuracies for dissociation reactions with late transition states can be directly related to chemisorption accuracies. Benchmarking studies of adsorption on transition metal surfaces demonstrate that accuracies can vary widely even within a given DFA class. ${ }^{9,28}$ As a result, systematic trends for GGA, metaGGA and hybrid functionals are difficult to determine for barriers on surfaces without an exhaustive study of various DFAs within each class. To illustrate this point, we compare $\mathrm{H}_{2}$ dissociation barriers for BEEF-vdW with another GGA, PBE. ${ }^{75}$ A comparison of PBE and BEEF$\mathrm{vdW}$ errors for chemisorption and barrier heights prediction is presented in the Supporting Information. Unlike BEEF-vdW, PBE underestimates barrier heights with an RMSE of $0.33 \mathrm{eV}$. While this is consistent with the fact that PBE is less accurate for chemisorption when compared with BEEF-vdW, ${ }^{9}$ it also demonstrates that barrier accuracies can vary within a particular DFA class.

DFA accuracies, based on the results in Table 3, also appear to be substrate-specific. Unfortunately, no direct comparison with chemisorption energies is available for dissociation of $\mathrm{CH}_{4}$, for which MS2 provides reliable barrier estimates for $\mathrm{Ru}(0001)$ and $\mathrm{Ni}(111)$ terrace sites. It is possible that the overbinding of the $\mathrm{H}$ atom by MS2 shown in Figure 3, is compensated by underbinding of the $\mathrm{CH}_{3}$ fragment, leading to accurate total chemisorption energies of the dissociated fragments, and hence reasonable barrier heights. Another possibility is that the agreement between various DFAs stems from a similar description of the gas phase $\mathrm{CH}_{4}$ molecule. The calculated errors in atomization energy per $\mathrm{C}-\mathrm{H}$ bond with BEEF-vdW, MS2, and HSE06 are $+0.03,-0.03$, and $+0.03 \mathrm{eV}$, respectively, which are negligible compared to errors in atomization energies of $\mathrm{H}_{2}$ or $\mathrm{N}_{2}$, which are $\pm 0.1 \mathrm{eV}$ or higher across the three functionals.

\section{CONCLUSIONS}

The SBH10 database of barrier heights along with the database of adsorption energies on transition metal surfaces, ${ }^{9,74}$ will be invaluable in designing and benchmarking density functionals for applications in surface chemistry and heterogeneous catalysis. The reference barriers are carefully chosen from thermal rate measurements, laser assisted associative desorption experiments, and molecular beam experiments combined with quantum dynamics models. The benchmark study shows that the BEEF-vdW GGA clearly outperforms the MS2 meta-GGA and HSE06 hybrid, in direct contradiction to the gas phase barrier accuracies of these functionals. This is because transition states for dissociative adsorption closely resemble final states, and errors in barrier heights mirror errors in chemisorption energies. In other words, these transition states closely interact with the surface, while gas phase transition states are relatively isolated species. Therefore, the key driver for functional accuracies for reactions on catalyst surfaces is the description of surface-adsorbate interactions, and not charge separation or self-interaction correction. 


\section{ASSOCIATED CONTENT}

\section{S Supporting Information}

The Supporting Information is available free of charge on the ACS Publications website at DOI: 10.1021/acs.jpcc.7b05677.

Details of model setup, calculation procedure, and transition state geometries calculated with the BEEF$\mathrm{vdW}$ functional, and contrast in the chemisorption errors and barriers for $\mathrm{H}_{2}$ dissociation with BEEF-vdW and PBE (PDF)

\section{AUTHOR INFORMATION}

\section{Corresponding Author}

*(J.K.N.) E-mail: norskov@stanford.edu.

\section{ORCID}

Shaama Mallikarjun Sharada: 0000-0001-7332-5373

Geert-Jan Kroes: 0000-0002-4913-4689

Notes

The authors declare no competing financial interest.

\section{ACKNOWLEDGMENTS}

This work is supported by a grant of the U.S. Department of Energy, Office of Science, Office of Basic Energy Sciences, to the SUNCAT Center for Interface Science and Catalysis.

\section{REFERENCES}

(1) Kohn, W.; Sham, L. J. Self-Consistent Equations Including Exchange and Correlation Effects. Phys. Rev. 1965, 140, A1133A1138.

(2) Kohn, W.; Becke, A. D.; Parr, R. G. Density Functional Theory of Electronic Structure. J. Phys. Chem. 1996, 100 (31), 12974-12980.

(3) Nørskov, J. K.; Abild-Pedersen, F.; Studt, F.; Bligaard, T. Density Functional Theory in Surface Chemistry and Catalysis. Proc. Natl. Acad. Sci. U. S. A. 2011, 108 (3), 937-943.

(4) Greeley, J.; Jaramillo, T. F.; Bonde, J.; Chorkendorff, I.; Nørskov, J. K. Computational High-throughput Screening of Electrocatalytic Materials for Hydrogen Evolution. Nat. Mater. 2006, 5, 909-913.

(5) Zhao, L. L.; Jensen, L.; Schatz, G. C. Pyridine- $\mathrm{Ag}_{20}$ Cluster: A Model System for Studying Surface-Enhanced Raman Scattering. J. Am. Chem. Soc. 2006, 128, 2911-2919.

(6) Liu, C.; Li, F.; Ma, L.-P.; Cheng, H.-M. Advanced Materials for Energy Storage. Adv. Mater. 2010, 22, E28-E62.

(7) Hammer, B.; Hansen, L. B.; Nørskov, J. K. Improved Adsorption Energetics within Density-Functional Theory using Revised PerdewBurke-Ernzerhof Functionals. Phys. Rev. B: Condens. Matter Mater. Phys. 1999, 59, 7413-7421.

(8) Wellendorff, J.; Lundgaard, K. T.; Møgelhøj, A.; Petzold, V.; Landis, D. D.; Nørskov, J. K.; Bligaard, T.; Jacobsen, K. W. Density Functionals for Surface Science: Exchange-Correlation Model Development with Bayesian Error Estimation. Phys. Rev. B: Condens. Matter Mater. Phys. 2012, 85 (23), 235149.

(9) Wellendorff, J.; Silbaugh, T. L.; Garcia-Pintos, D.; Nørskov, J. K.; Bligaard, T.; Studt, F.; Campbell, C. T. A Benchmark Database for Adsorption Bond Energies to Transition Metal Surfaces and Comparison to Selected DFT Functionals. Surf. Sci. 2015, 640, 36-44.

(10) Hensley, A. J. R.; Ghale, K.; Rieg, C.; Dang, T.; Anderst, E.; Studt, F.; Campbell, C. T.; McEwen, J.-S.; Xu, Y. DFT-Based Method for More Accurate Adsorption Energies: An Adaptive Sum of Energies from RPBE and vdW Density Functionals. J. Phys. Chem. C 2017, 121, 4937-4945.

(11) Perdew, J. P. Jacob's Ladder of Density Functional Approximations for the Exchange-Correlation Energy. AIP Conf. Proc. 2000, 577, 1-20.

(12) Mori-Sánchez, P.; Cohen, A. J.; Yang, W. Many-electron Selfinteraction Error in Approximate Density Functionals. J. Chem. Phys. 2006, 125, 201102.
(13) Perdew, J. P. Density Functional Methods In Physics; Dreizler, R M., da Providência, J., Eds.; Springer: New York, 1985; pp 265-308.

(14) Becke, A. D. Density-Functional Thermochemistry. III. The Role of Exact Exchange. J. Chem. Phys. 1993, 98 (7), 5648.

(15) Lynch, B. J.; Truhlar, D. G. How Well Can Hybrid Density Functional Methods Predict Transition State Geometries and Barrier Heights? J. Phys. Chem. A 2001, 105 (13), 2936-2941.

(16) Zheng, J.; Zhao, Y.; Truhlar, D. G. The DBH24/08 Database and Its Use to Assess Electronic Structure Model Chemistries for Chemical Reaction Barrier Heights. J. Chem. Theory Comput. 2009, 5, $808-821$.

(17) Zhao, Y.; Gónzalez-García, N.; Truhlar, D. G. Benchmark Database of Barrier Heights for Heavy Atom Transfer, Nucleophilic Substitution, Association, and Unimolecular Reactions and Its Use to Test Theoretical Methods. J. Phys. Chem. A 2005, 109, 2012-2018.

(18) Raghavachari, K.; Trucks, G. W.; et al. A Fifth-Order Perturbation Comparison of Electron Correlation Theories. Chem. Phys. Lett. 1989, 157, 479-483.

(19) Kroes, G. J. Toward a Database of Chemically Accurate Barrier Heights for Reactions of Molecules with Metal Surfaces. J. Phys. Chem. Lett. 2015, 6 (20), 4106-4114.

(20) Kroes, G. J. Towards Chemically Accurate Simulation of Molecule-Surface Reactions. Phys. Chem. Chem. Phys. 2012, 14, 14966-14981.

(21) Díaz, C.; Olsen, R. A.; Auerbach, D. J.; Kroes, G. J. Sixdimensional Dynamics Study of Reactive and Non Reactive Scattering of $\mathrm{H}_{2}$ from $\mathrm{Cu}(111)$ using a Chemically Accurate Potential Energy Surface. Phys. Chem. Chem. Phys. 2010, 12, 6499-6519.

(22) Diekhöner, L.; Mortensen, H.; Baurichter, A.; Luntz, A. C. Laser Assisted Associative Desorption of $\mathrm{N} 2$ and $\mathrm{CO}$ from $\mathrm{Ru}(0001)$. J. Chem. Phys. 2001, 115 (7), 3356-3373.

(23) Dahl, S.; Logadottir, A.; Egeberg, R. C.; Larsen, J. H.; Chorkendorff, I.; Törnqvist, E.; Nørskov, J. K. Role of Steps in Activation on $\mathrm{Ru}(0001)$. Phys. Rev. Lett. 1999, 83 (9), 1814-1817.

(24) Kresse, G.; Hafner, J. Ab Initio Molecular Dynamics for Liquid Metals. Phys. Rev. B: Condens. Matter Mater. Phys. 1993, 47, 558.

(25) Kresse, G.; Hafner, J. Ab Initio Molecular-Dynamics Simulation of the Liquid-Metal-Amorphous-Semiconductor Transition in Germanium. Phys. Rev. B: Condens. Matter Mater. Phys. 1994, 49, 14251.

(26) Kresse, G.; Furthmüller, J. Efficiency of Ab-Initio Total Energy Calculations for Metals and Semiconductors Using a Plane-Wave Basis Set. Comput. Mater. Sci. 1996, 6, 15.

(27) Kresse, G.; Furthmüller, J. Efficient Iterative Schemes for $\mathrm{Ab}$ Initio Total-Energy Calculations Using a Plane-Wave Basis Set. Phys. Rev. B: Condens. Matter Mater. Phys. 1996, 54, 11169.

(28) Hensley, A. J. R.; Ghale, K.; Rieg, C.; Dang, T.; Anderst, E. S.; Studt, F.; Campbell, C. T.; McEwen, J.-S.; Xu, Y. A DFT-Based Method for More Accurate Adsorption Energies: An Adaptive Sum of Energies from RPBE and vdW Density Functionals. J. Phys. Chem. C 2017, 121, 4937-4945.

(29) Lee, K.; Murray, E. D.; Kong, L.; Lundqvist, B. I.; Langreth, D. C. Higher-Accuracy van der Waals Density Functional. Phys. Rev. B: Condens. Matter Mater. Phys. 2010, 82, 081101.

(30) Román-Pérez, G.; Soler, J. M. Efficient Implementation of a van der Waals Density Functional: Application to Double-Wall Carbon Nanotubes. Phys. Rev. Lett. 2009, 103, 096102.

(31) Sun, J.; Xiao, B.; Fang, Y.; Haunschild, R.; Hao, P.; Ruzsinszky, A.; Csonka, G. I.; Scuseria, G. E.; Perdew, J. P. Density Functionals That Recognize Covalent, Metallic, and Weak Bonds. Phys. Rev. Lett. 2013, 111 (10), 106401.

(32) Monkhorst, H. J. Hartree-Fock Density of States for Extended Systems. Phys. Rev. B: Condens. Matter Mater. Phys. 1979, 20, 15041513.

(33) Stroppa, A.; Termentzidis, K.; Paier, J.; Kresse, G.; Hafner, J. CO Adsorption on Metal Surfaces: A Hybrid Functional Study with Plane-Wave Basis Set. Phys. Rev. B: Condens. Matter Mater. Phys. 2007, $76,195440$. 
(34) Stroppa, A.; Kresse, G. The Shortcomings of Semi-Local and Hybrid Functionals: What we can Learn from Surface Science Studies. New J. Phys. 2008, 10, 063020.

(35) Krukau, A. V.; Vydrov, O. A.; Izmaylov, A. F.; Scuseria, G. E. Influence of the Exchange Screening Parameter on the Performance of Screened Hybrid Functionals. J. Chem. Phys. 2006, 125, 224106.

(36) Sementa, L.; Wijzenbroek, M.; Van Kolck, B. J.; Somers, M. F.; Al-Halabi, A.; Busnengo, H. F.; Olsen, R. A.; Kroes, G. J.; Rutkowski, M.; Thewes, C.; et al. Reactive Scattering of $\mathrm{H}_{2}$ from $\mathrm{Cu}(100)$ : Comparison of Dynamics Calculations Based on the Specific Reaction Parameter Approach to Density Functional Theory with Experiment. J. Chem. Phys. 2013, 138, 044708.

(37) Kroes, G. J.; Díaz, C. Quantum and Classical Dynamics of Reactive Scattering of $\mathrm{H} 2$ from Metal Surfaces. Chem. Soc. Rev. 2016, $45,3658-3700$.

(38) Luntz, A. C.; Persson, M. How Adiabatic Is Activated Adsorption/ Associative Desorption? J. Chem. Phys. 2005, 123, 074704.

(39) Kroes, G. J. Six-dimensional Quantum Dynamics of Dissociative Chemisorption of $\mathrm{H}_{2}$ on Metal Surfaces. Prog. Surf. Sci. 1999, 60, 185 .

(40) Díaz, C.; Pijper, E.; Olsen, R. A.; Busnengo, H. F.; Auerbach, D. J.; Kroes, G. J. Chemically Accurate Simulation of a Prototypical Surface Reaction: $\mathrm{H} 2$ Dissociation on $\mathrm{Cu}(111)$. Science 2009, 326, 832-834.

(41) Rasmussen, P. B.; Holmblad, P. M.; Christoffersen, H.; Taylor, P. a.; Chorkendorff, I. Dissociative Adsorption of Hydrogen on $\mathrm{Cu}(100)$ at Low Temperatures. Surf. Sci. 1993, 287-288, 79-83.

(42) Campbell, J. M.; Campbell, C. T. The Dissociative Adsorption of $\mathrm{H}_{2}$ and $\mathrm{D}_{2}$ on $\mathrm{Cu}(110)$ : Activation Barriers and Dynamics. Surf. Sci. 1991, 259, 1-17.

(43) Wijzenbroek, M.; Kroes, G. J. The Effect of the ExchangeCorrelation Functional on $\mathrm{H}_{2}$ Dissociation on $\mathrm{Ru}(0001)$. J. Chem. Phys. 2014, 140, 084702.

(44) Luntz, A. C.; Persson, M.; Wagner, S.; Frischkorn, C.; Wolf, M. Femtosecond Laser Induced Associative Desorption of $\mathrm{H}_{2}$ from $\mathrm{Ru}(0001)$ : Comparison Of "first Principles" theory with Experiment. J. Chem. Phys. 2006, 124, 244702.

(45) Vincent, J. K.; Olsen, R. A.; Kroes, G. J.; Baerends, E. J. Dissociative Chemisorption of $\mathrm{H}_{2}$ on $\mathrm{Pt}(111)$ : Isotope Effect and Effects of the Rotational Distribution and Energy Dispersion. Surf. Sci. 2004, 573, 433-445.

(46) Nour Ghassemi, E.; Wijzenbroek, M.; Somers, M. F.; Kroes, G.J. Chemically Accurate Simulation of Dissociative Chemisorption of $\mathrm{D}_{2}$ on $\mathrm{Pt}(111)$. Chem. Phys. Lett. 2017, 683, 329-335.

(47) Schlögl, R. Catalytic Synthesis of Ammonia - A "Never-Ending Story"? Angew. Chem., Int. Ed. 2003, 42 (18), 2004-2008.

(48) Dahl, S.; Törnqvist, E.; Chorkendorff, I. Dissociative Adsorption of $\mathrm{N}_{2}$ on $\mathrm{Ru}(0001)$ : A Surface Reaction Totally Dominated by Steps. J. Catal. 2000, 192 (2), 381-390.

(49) Dahl, S.; Taylor, P. A.; Törnqvist, E.; Chorkendorff, I. The Synthesis of Ammonia over a Ruthenium Single Crystal. J. Catal. 1998, 178 (2), 679-686.

(50) Díaz, C.; Vincent, J. K.; Krishnamohan, G. P.; Olsen, R. A.; Kroes, G. J.; Honkala, K.; Norskov, J. K. Reactive and Nonreactive Scattering of $\mathrm{N}_{2}$ from $\mathrm{Ru}(0001)$ : A Six-Dimensional Adiabatic Study. J. Chem. Phys. 2006, 125, 114706.

(51) Shakouri, K.; Behler, J.; Meyer, J.; Kroes, G.-J. Accurate Neural Network Description of Surface Phonons in Reactive Gas-Surface Dynamics: $\mathrm{N}_{2}+\mathrm{Ru}(0001)$. J. Phys. Chem. Lett. 2017, 8, 2131-2136.

(52) Nave, S.; Jackson, B. Methane Dissociation on Ni (111) and Pt (111): Energetic and Dynamical Studies. J. Chem. Phys. 2009, 130, 054701.

(53) Jackson, B.; Nave, S. The Dissociative Chemisorption of Methane on $\mathrm{Ni}(111)$ : The Effects of Molecular Vibration and Lattice Motion. J. Chem. Phys. 2013, 138, 174705.

(54) Egeberg, R. C.; Ullmann, S.; Alstrup, I.; Mullins, C. B.; Chorkendorff, I. Dissociation of $\mathrm{CH} 4$ on $\mathrm{Ni}(111)$ and $\mathrm{Ru}(0001)$. Surf. Sci. 2002, 497, 183-193.
(55) Nattino, F.; Migliorini, D.; Kroes, G.-J.; Dombrowski, E.; High, E. A.; Killelea, D. R.; Utz, A. L. Chemically Accurate Simulation of a Polyatomic Molecule-Metal Surface Reaction. J. Phys. Chem. Lett. 2016, 7, 2402-2406.

(56) Abild-Pedersen, F.; Lytken, O.; Engbæk, J.; Nielsen, G.; Chorkendorff, I.; Nørskov, J. K. Methane Activation on $\mathrm{Ni}(111)$ : Effects of Poisons and Step Defects. Surf. Sci. 2005, 590 (2-3), 127137.

(57) Ølgaard Nielsen, B.; Luntz, A. C.; Holmblad, P. M.; Chorkendorff, I. Activated Dissociative Chemisorption of Methane on Ni(100): A Direct Mechanism under Thermal Conditions? Catal. Lett. 1995, 32 (1-2), 15-30.

(58) Luntz, a. C. $\mathrm{CH}_{4}$ Dissociation on $\mathrm{Ni}(100)$ : Comparison of a Direct Dynamical Model to Molecular Beam Experiments. J. Chem. Phys. 1995, 102, 8264.

(59) Larsen, J. H.; Holmblad, P. M.; Chorkendorff, I. Dissociative Sticking of $\mathrm{CH}_{4}$ on $\mathrm{Ru}(0001)$. J. Chem. Phys. 1999, 110 (5), 2637.

(60) Mortensen, H.; Diekhöner, L.; Baurichter, A.; Luntz, A. C. $\mathrm{CH}_{4}$ Dissociation on $\mathrm{Ru}(0001)$ : A View from Both Sides of the Barrier. J. Chem. Phys. 2002, 116 (13), 5781-5794.

(61) Andersson, M. P.; Abild-Pedersen, F.; Remediakis, I. N.; Bligaard, T.; Jones, G.; Engbæk, J.; Lytken, O.; Horch, S.; Nielsen, J. H.; Sehested, J.; et al. Structure Sensitivity of the Methanation Reaction: H2-Induced CO Dissociation on Nickel Surfaces. J. Catal. 2008, 255, 6-19.

(62) Migliorini, D.; Chadwick, H.; Nattino, F.; Gutiérrez-González, A.; Dombrowski, E.; High, E. A.; Guo, H.; Utz, A. L.; Jackson, B.; Beck, R. D.; Kroes, G.-J. Surface reaction barriometry: methane dissociation on flat and stepped transition metal surfaces. J. Phys. Chem. Lett. 2017, 8, 4177-4182.

(63) Mortensen, H.; Diekhöner, L.; Baurichter, A.; Jensen, E.; Luntz, A. C. Dynamics of Ammonia Decomposition on $\mathrm{Ru}(0001)$. J. Chem. Phys. 2000, 113 (16), 6882-6887.

(64) Hundt, P. M.; Jiang, B.; van Reijzen, M. E.; Guo, H.; Beck, R. D. Vibrationally Promoted Dissociation of Water on $\mathrm{Ni}(111)$. Science 2014, 344, 504-507.

(65) Rettner, C. T.; Michelsen, H. A.; Auerbach, D. J. QuantumState-Specific Dynamics of the Dissociative Adsorption and Associative Desorption of $\mathrm{H}_{2}$ at a $\mathrm{Cu}(111)$ Surface. J. Chem. Phys. 1995, 102, 4625.

(66) Berger, H. F.; Leisch, M.; Winkler, A.; Rendulic, K. D. A Search for Vibrational Contributions to the Activated Adsorption of $\mathrm{H}_{2}$ on Copper. Chem. Phys. Lett. 1990, 175, 425-428.

(67) Anger, G.; Winkler, A.; Rendulic, K. D. Adsorption and Desorption Kinetics in the Systems $\mathrm{H}_{2} / \mathrm{Cu}(111), \mathrm{H}_{2} / \mathrm{Cu}(110)$, and $\mathrm{H}_{2} / \mathrm{Cu}(100)$. Surf. Sci. 1989, 220, 1-17.

(68) Luntz, A. C.; Brown, J. K.; Williams, M. D. Molecular Beam Studies of $\mathrm{H}_{2}$ and $\mathrm{D}_{2}$ Dissociative Chemisorption on $\mathrm{Pt}(111)$. J. Chem. Phys. 1990, 93, 5240.

(69) Groot, I. M. N.; Ueta, H.; van der Niet, M. J. T. C.; Kleyn, A. W.; Juurlink, L. B. F. Supersonic Molecular Beam Studies of Dissociative Adsorption of $\mathrm{H}_{2}$ on $\mathrm{Ru}(0001)$. J. Chem. Phys. 2007, $127,244701$.

(70) Diekhöner, L.; Mortensen, H.; Baurichter, A.; Jensen, E.; Petrunin, V. V.; Luntz, A. C. $\mathrm{N}_{2}$ Dissociative Adsorption on $\mathrm{Ru}(0001)$ : The Role of Energy Loss. J. Chem. Phys. 2001, 115, 9028.

(71) Nieto, P.; Pijper, E.; Barredo, D.; Laurent, G.; Olsen, R. A.; Baerends, E.-J.; Kroes, G.-J.; Farias, D. Reactive and Nonreactive Scattering of $\mathrm{H}_{2}$ from a Metal Surface Is Electronically Adiabatic. Science 2006, 312, 86-89.

(72) Janesko, B. G.; Scuseria, G. E. Hartree-Fock Orbitals Significantly Improve the Reaction Barrier Heights Predicted by Semilocal Density Functionals. J. Chem. Phys. 2008, 128, 244112.

(73) Medford, A. J.; Wellendorff, J.; Vojvodic, A.; Studt, F.; Abildpedersen, F.; Jacobsen, K. W.; Bligaard, T.; et al. Assessing the Reliability of Calculated Catalytic Ammonia Synthesis Rates. Science 2014, 345, 197-200.

(74) Mallikarjun Sharada, S.; Karlsson, R. K. B.; Voss, J.; Bligaard, T. Manuscript in preparation. 
(75) Perdew, J.; Burke, K.; Ernzerhof, M. Generalized Gradient Approximation Made Simple. Phys. Rev. Lett. 1996, 77 (18), 38653868. 